\title{
A STRUCTURAL CRITERION FOR THE EXISTENCE OF INFINITE CENTRAL $\Lambda(p)$ SETS
}

\author{
KATHRYN E. HARE AND DAVID C. WILSON
}

\begin{abstract}
We classify the compact, connected groups which have infinite central $\Lambda(p)$ sets, arithmetically characterize central $\Lambda(p)$ sets on certain product groups, and give examples of $\Lambda(p)$ sets which are non-Sidon and have unbounded degree. These sets are intimately connected with Figà-Talamanca and Rider's examples of Sidon sets, and stem from the existence of families of tensor product representations of almost simple Lie groups whose decompositions into irreducibles are rank-independent.
\end{abstract}

\section{INTRODUCTION}

In this paper we arithmetically characterize central $\Lambda(p)$ sets on products of compact connected almost simple Lie groups, and we give the first published (so far as we are aware) examples of non-Sidon sets which are $\Lambda(p)$ sets for all $p \in(2, \infty)$ and which contain representations of arbitrarily large degree.

Unlike the abelian situation, there are examples (cf. [6]) of infinite nonabelian compact connected groups which admit no infinite central $\Lambda(4)$ sets. We will classify those compact connected groups which do admit infinite central $\Lambda(4)$ sets, and we prove that every infinite local $\Lambda(p)$ set contains an infinite $\Lambda(p)$ set.

The problem of finding non-Sidon sets on compact abelian groups, which were $\Lambda(p)$ sets for all $p \in(2, \infty)$, was solved by Bonami [2] by taking $m$-fold products of dissociate sets. Our examples are similar: we take the irreducible subrepresentations of $m$-fold tensor products of certain independent sets, christened FTR sets in [5]. These sets were Figà-Talamanca and Rider's examples of Sidon sets with unbounded degree [8]. Ideas from both Bonami's and FigàTalamanca and Rider's work can be found in our proofs.

\section{Preliminaries}

Let $G$ be a compact group. The dual object $\widehat{G}$ of $G$ will be taken to be a maximal set of pairwise inequivalent continuous unitary irreducible representations of $G$. If $\sigma$ is an irreducible component of the continuous unitary

Received by the editors March 13, 1991.

1980 Mathematics Subject Classification (1985 Revision). Primary 43A46; Secondary 43A80, 22E46.

Key words and phrases. $\Lambda(p)$ set, lacunary set, representations of compact groups.

This research was carried out while the first author enjoyed the hospitality of The University of New South Wales and was partially supported by the NSERC. 
representation $\gamma$ of $G$ we write $\sigma \leq \gamma$, and we denote by $d(\gamma)$ its degree. Since we consider only continuous homomorphisms, we will henceforth take "continuous" to be understood. The representation obtained by taking the $m$ fold tensor product of $\sigma$ with itself will be denoted $\sigma^{m}$ (and of course we take $\sigma^{0}$ to be the trivial representation); we also write $\sigma^{-m}$ for $(\bar{\sigma})^{m}$. If $E \subseteq \widehat{G}$ then we write

$$
E^{m}=\left\{\sigma \in \widehat{G}: \sigma \leq \gamma_{1} \otimes \cdots \otimes \gamma_{m}, \gamma_{1}, \ldots, \gamma_{m} \in E\right\}
$$

(see $[9,27.35])$. If $\widehat{G}$ contains at most finitely many elements of each degree we call $G$ tall. By $\mathscr{T}_{E}(G)$ we denote the space of trigonometric polynomials $f$ on $G$ whose Fourier transforms $\widehat{f}$ are supported by $E \subseteq \widehat{G}$, and by $\mathscr{T}_{E}{ }^{z}(G)$ we denote the subspace of central trigonometric polynomials.

Let $p \in(2, \infty)$. A subset $E$ of $\widehat{G}$ is called a (central) $\Lambda(p)$ set if there is a constant $C_{p}$ such that $\|f\|_{p} \leq C_{p}\|f\|_{2}$ whenever $f \in \mathscr{T}_{E}(G)\left(f \in \mathscr{T}_{E}^{z}(G)\right)$, and $E$ is called a local (central) $\Lambda(p)$ set if the inequality $\|\operatorname{tr} A \sigma\|_{p} \leq C_{p}\|\operatorname{tr} A \sigma\|_{2}$ holds for all $\sigma \in E$ and (central) matrices $A$. The least such constants $C_{p}$ are called the (central) $\Lambda(p)$ constants or local (central) $\Lambda(p)$ constants accordingly, and will be denoted by $\eta_{p}(E) \quad\left(\zeta_{p}(E)\right)$ and $\eta_{p}^{0}(E) \quad\left(\zeta_{p}^{0}(E)\right)$. For standard results on $\Lambda(p)$ sets and equivalent characterizations we refer the reader to $[9, \S 37]$.

\section{LIE THEORY AND TENSOR PRODUCT DECOMPOSITIONS}

We fix in this section certain notation which will be used throughout the paper, and summarize some of the representation theory of compact groups which is required.

If $\widetilde{G}$ is a simply-connected compact Lie group then we denote by $\Phi$ the corresponding root system, by $\Lambda$ the weight lattice, and we fix a base $\Delta=$ $\left\{\alpha_{1}, \ldots, \alpha_{l}\right\}$ ( $l$ the rank of $\widetilde{G}$ ) for $\Phi$. We write $\Phi^{+}$for the positive roots and $\Lambda^{+}$for the positive cone of $\Lambda$ (relative to $\Delta$ ), with the fundamental weights, denoted $\lambda_{1}, \ldots, \lambda_{l}$, ordered as in [17]. The fundamental weights are an integral basis for $\Lambda$, dual to $\Delta$ in that $\left\langle\lambda_{j}, \alpha_{k}\right\rangle=2\left(\lambda_{j}, \alpha_{k}\right) /\left(\alpha_{k}, \alpha_{k}\right)=\delta_{j k}$. We put $\delta=\sum_{j=1}^{l} \lambda_{j} ; \delta$ is also half the sum of the positive roots. A partial order is defined on $\Lambda$ by $\mu \prec \lambda$ iff $\lambda-\mu$ is a nonnegative integral sum of positive roots. The Weyl group will be denoted by $\mathscr{W}$, and the weights of $\lambda \in \Lambda^{+}$by $\Pi(\lambda)=\{\mu \in \Lambda: w \mu \prec \lambda$ for all $w \in \mathscr{W}\}$.

Let $G$ be a compact connected almost simple Lie group, and let $\widetilde{G}$ be its simply-connected covering group. Then the dual object of $G$ may be identified (via the highest weight of each representation) with the positive cone of a sublattice of $\Lambda$ containing the root lattice. We abuse notation somewhat and write $\lambda$ for the irreducible representation of $\widetilde{G}$ (or $G$, depending on the context) whose highest weight is $\lambda \in \Lambda^{+}$. The symbol $\varsigma$ will be used to denote the representation of $\widetilde{G}$ whose highest weight is $\lambda_{1}$, if $G$ is not exceptional (it is sometimes convenient to consider types $B_{2}$ and $D_{4}$ exceptional), or the trivial representation otherwise.

One reason for the importance of tensor product decompositions lies in the simple observation that for $G$ any compact group and $\sigma \in \widehat{G}$, the central $\Lambda(4)$ constant of the singleton $\{\sigma\}$ is determined by the number of irreducible com- 
ponents of $\sigma \otimes \sigma$ (or $\sigma \otimes \bar{\sigma}$ ), counted by squared multiplicities (see (5.2) below). For $G$ almost simple Lie, there are formulæ which give explicit decompositions of tensor products, such as that due to Brauer-Klimyk:

(3.1) Theorem [13, Problem IV.16]. Let $\lambda, \lambda^{\prime} \in \Lambda^{+}$. Denote by $m_{\lambda}(\mu)$ the multiplicity of $\mu \in \Lambda$ in $\Pi(\lambda)$ and by $\tilde{\mu}$ the (unique) element of $\Lambda^{+} \mathscr{W}$ conjugate to $\mu$ [11, Lemma 13.2.A]. Define

$$
\operatorname{sgn} \mu= \begin{cases}0 & \text { if } \mu \text { is fixed by some } w \neq 1 \in \mathscr{W}, \\ \operatorname{det} w & \text { otherwise, where } w \mu=\widetilde{\mu} .\end{cases}
$$

Then

$$
\operatorname{tr}\left(\lambda \otimes \lambda^{\prime}\right)=\sum_{\mu \in \Pi(\lambda)} m_{\lambda}(\mu) \operatorname{sgn}\left(\mu+\lambda^{\prime}+\delta\right) \operatorname{tr}\left(\left(\mu+\lambda^{\prime}+\delta\right)^{\sim}-\delta\right) .
$$

Neither this formula nor that of Steinberg (see [11, Theorem 24.4]) gives a straightforward way to count the number of components occurring in the decomposition, because of cancellation. However, it is possible to determine the form of a few special summands, and hence obtain a lower bound for the number of components.

(3.2) Lemma [7, Lemma 3.8]. Let $\lambda, \lambda^{\prime} \in \Lambda^{+}$. Suppose that $k \in \mathbb{N}$ satisfies $k \leq \min \left(\left\langle\lambda, \alpha_{i}\right\rangle,\left\langle\lambda^{\prime}, \alpha_{i}\right\rangle\right)$ for some $i \in\{1, \ldots, l\}$. Then $\mu=\lambda+\lambda^{\prime}-k \alpha_{i} \in$ $\Lambda^{+}$, and $\mu$ occurs with multiplicity one in $\lambda \otimes \lambda^{\prime}$.

(3.3) Lemma. Let $\lambda, \lambda^{\prime} \in \Lambda^{+}$and suppose $\mu=w(\lambda)$, some $w \in \mathscr{W}$, satisfies $\mu+\lambda^{\prime} \in \Lambda^{+}$. Then $\mu+\lambda^{\prime}$ occurs with multiplicity one in $\lambda \otimes \lambda^{\prime}$.

Proof. From Theorem (3.1) it suffices to prove that the only $\nu \in \Pi(\lambda)$ for which $\nu^{\prime}=\nu+\lambda^{\prime}+\delta$ is $\mathscr{W}$-conjugate to $\mu^{\prime}=\mu+\lambda^{\prime}+\delta$ is $\nu=\mu$. So suppose that $\nu^{\prime}$ is $\mathscr{W}$-conjugate to $\mu^{\prime}$.

Since $\mu^{\prime} \in \Lambda^{+}$, it follows [11, Lemma 13.2.A] that $\nu^{\prime} \prec \mu^{\prime}$ and hence that $\mu-\nu=\rho^{\prime}$ where $\rho^{\prime}$ is a nonnegative integral sum of positive roots. Since the Weyl group preserves the inner product, we also have

$$
\left(\nu^{\prime}, \nu^{\prime}\right)=\left(\mu^{\prime}, \mu^{\prime}\right) \text {. }
$$

Now $[11,13.4] \nu \in \Pi(\lambda)$ means that $w^{\prime}(\nu)=\lambda-\rho \in \Lambda^{+}$for some $w^{\prime} \in \mathscr{W}$ and some nonnegative integral sum $\rho$ of positive roots. Hence

$$
\begin{aligned}
\left(\nu^{\prime}, \nu^{\prime}\right) & =\left(w^{\prime}\left(\nu^{\prime}\right), w^{\prime}\left(\nu^{\prime}\right)\right) \\
& =\left(\lambda-\rho+w^{\prime}\left(\lambda^{\prime}+\delta\right), \lambda-\rho+w^{\prime}\left(\lambda^{\prime}+\delta\right)\right) \\
& =\left(\lambda+w^{\prime}\left(\lambda^{\prime}+\delta\right), \lambda+w^{\prime}\left(\lambda^{\prime}+\delta\right)\right)+(\rho, \rho)-2\left(\rho, \lambda+w^{\prime}\left(\lambda^{\prime}+\delta\right)\right) \\
& =\left(\mu+w w^{\prime}\left(\lambda^{\prime}+\delta\right), \mu+w w^{\prime}\left(\lambda^{\prime}+\delta\right)\right) \\
& -(2 \lambda-\rho, \rho)+2\left(-\rho, w^{\prime}\left(\lambda^{\prime}+\delta\right)\right) \\
& \leq\left(\mu^{\prime}, \mu^{\prime}\right)-2\left(\mu, \lambda^{\prime}+\delta-w w^{\prime}\left(\lambda^{\prime}+\delta\right)\right)+2\left(-\rho, w^{\prime}\left(\lambda^{\prime}+\delta\right)\right)
\end{aligned}
$$

(using the fact that $(2 \lambda-\rho, \rho) \geq 0$ since $\left.2 \lambda-\rho \in \Lambda^{+}\right)$

$$
\begin{aligned}
& =\left(\mu^{\prime}, \mu^{\prime}\right)-2\left(\mu, \lambda^{\prime}+\delta\right)+2\left(\mu, w w^{\prime}\left(\lambda^{\prime}+\delta\right)\right)+2\left(-\rho, w^{\prime}\left(\lambda^{\prime}+\delta\right)\right) \\
& =\left(\mu^{\prime}, \mu^{\prime}\right)-2\left(\mu, \lambda^{\prime}+\delta\right)+2\left(\lambda-\rho, w^{\prime}\left(\lambda^{\prime}+\delta\right)\right)
\end{aligned}
$$$$
=\left(\mu^{\prime}, \mu^{\prime}\right)-2\left(\mu-\nu, \lambda^{\prime}+\delta\right) \text {, }
$$ 
so that $\left(\mu-\nu, \lambda^{\prime}+\delta\right) \leq 0$ is necessary for $(*)$. But since $\left(\alpha, \lambda^{\prime}+\delta\right)>0$ for each $\alpha \in \Phi^{+}$and $\mu-\nu=\rho^{\prime}$, this forces $\mu=\nu$.

(3.4) Lemma. Suppose $\lambda=\sum_{k=1}^{l} m_{k} \lambda_{k} \in \Lambda^{+}$where $\Phi$ is of type $A_{l}, B_{l}, C_{l}$, or $D_{l}$. Then $\lambda \otimes \bar{\lambda}$ has at least $N+1$ components, where

$$
N=\left\{\begin{array}{l}
\max \left\{k: k \leq(l+1) / 2 \text { and } m_{k}+m_{l+1-k} \neq 0\right\} \text { for type } A_{l}, \\
\max \left\{k: m_{k} \neq 0\right\} \text { for types } B_{l} \text { and } C_{l}, \\
\max \left\{k: m_{k} \neq 0\right\} \text { for type } D_{l}, \text { if this is less than } l-1 \\
{[l / 2] \text { otherwise. }}
\end{array}\right.
$$

Proof. We deal first with the case $A_{l}$. Note that $\bar{\lambda}=\sum_{j=1}^{l} m_{l+1-j} \lambda_{j}$, and that [17; 11, Table 11.4.1] for $1 \leq i \leq j \leq l$ we have $\beta_{i j}=\sum_{k=i}^{j} \alpha_{k}=$ $-\lambda_{i-1}+\lambda_{i}+\lambda_{j}-\lambda_{j+1} \in \Phi^{+}$(where we set $\lambda_{0}=\lambda_{l+1}=0$ ). Write $w_{i j}$ for the reflection corresponding to $\beta_{i j}$ :

$$
w_{i j}(\lambda)=\lambda-\sum_{k=i}^{j} m_{k} \beta_{i j}
$$

It is straightforward, albeit tedious, to prove by induction that for $1 \leq i \leq N$,

$$
\begin{aligned}
\bar{\lambda}+ & w_{i, l+1-i} w_{i+1, l-i} \cdots w_{N, l+1-N}(\lambda) \\
& =\sum_{k=1}^{i-2}\left(m_{k}+m_{l+1-k}\right)\left(\lambda_{k}+\lambda_{l+1-k}\right)+\sum_{k=i-1}^{N}\left(m_{k}+m_{l+1-k}\right)\left(\lambda_{i-1}+\lambda_{l+2-i}\right) .
\end{aligned}
$$

Since each of these belongs to $\Lambda^{+}$, by Lemma (3.3), each occurs in $\lambda \otimes \bar{\lambda}$; furthermore, they are clearly distinct. The other guaranteed component is $\bar{\lambda}+\lambda$, giving at least $N+1$ components as claimed.

The argument for the remaining types is similar. For type $B_{l}$ we have $\lambda=\bar{\lambda}$; $\beta_{i}=\sum_{k=i}^{l} \alpha_{k} \in \Phi^{+}$and $\beta_{i}=-\lambda_{i-1}+\lambda_{i}, 1 \leq i<l$, while $\beta_{l}=-\lambda_{l-1}+2 \lambda_{l}$. Writing $w_{i}(\lambda)=\lambda-\left(m_{l}+2 \sum_{k=i}^{l-1} m_{k}\right) \beta_{i}$ we again prove by induction that for $1 \leq i \leq l$,

$$
\lambda+w_{i} w_{i+1} \cdots w_{l}(\lambda)=2 \sum_{k=1}^{i-2} m_{k} \lambda_{k}+\left(m_{l}+2 \sum_{k=i-1}^{l-1} m_{k}\right) \lambda_{i-1},
$$

and these are distinct elements of $\Lambda^{+}$for $1 \leq i \leq N$; observe also that $2 \lambda$ occurs in $\lambda \otimes \lambda$.

For type $C_{l}$ we have $\lambda=\bar{\lambda} ; \beta_{i}=\alpha_{l}+2 \sum_{k=i}^{l-1} \alpha_{k}=-2 \lambda_{i-1}+2 \lambda_{i} \in \Phi^{+}$for $1 \leq i \leq l$, and writing $w_{i}(\lambda)=\lambda-\sum_{k=i}^{l} m_{k} \beta_{i}$ we again prove by induction that for $1 \leq i \leq N$,

$$
\lambda+w_{i} w_{i+1} \cdots w_{l}(\lambda)=2 \sum_{k=1}^{i-2} m_{k} \lambda_{k}+2 \sum_{k=i}^{N} m_{k} \lambda_{i-1} .
$$

As before, $2 \lambda$ also occurs in $\lambda \otimes \lambda$.

Type $D_{l}$ is a little more complicated. If $l$ is even we have $\lambda=\bar{\lambda}$, but if $l$ is odd, then $\bar{\lambda}=\sum_{k=1}^{l-1} m_{k} \lambda_{k}+m_{l} \lambda_{l-1}+m_{l-1} \lambda_{l}$. For $1 \leq i<l$ we 
have $\beta_{i}=\alpha_{l-1}+\sum_{k=i}^{l-2} \alpha_{k}$ and $\beta_{i}^{\prime}=\alpha_{l}+\sum_{k=i}^{l-2} \alpha_{k} \in \Phi^{+}$. Writing $w_{i}$ for the composition of the reflections corresponding to $\beta_{i}$ and $\beta_{i}^{\prime}$ we have

$$
\begin{gathered}
w_{l-1}(\lambda)=\sum_{k=1}^{l-3} m_{k} \lambda_{k}+\left(m_{l-2}+m_{l-1}+m_{l}\right) \lambda_{l-2}-\sum_{k=l-1}^{l} m_{k} \lambda_{k} \\
w_{i}(\lambda)=\sum_{k=1}^{i-2} m_{k} \lambda_{k}+\sum_{k=i+1}^{l-2} m_{k} \lambda_{k}+\left(m_{i-1}+m_{l-1}+m_{l}+2 \sum_{k=i}^{l-2} m_{k}\right) \lambda_{i-1} \\
-\left(m_{i}+m_{l-1}+m_{l}+2 \sum_{k=i+1}^{l-2} m_{k}\right) \lambda_{i}+m_{l} \lambda_{l-1}+m_{l-1} \lambda_{l} .
\end{gathered}
$$

Once again, by induction we prove that for $1 \leq i<l$,

$$
\begin{aligned}
\lambda+w_{i} w_{i+1} \cdots w_{l-1}(\lambda)= & 2 \sum_{k=1}^{i-2} m_{k} \lambda_{k}+\left(m_{l-1}+m_{l}+2 \sum_{k=i-1}^{l-2} m_{k}\right) \lambda_{i-1} \\
& +\left(m_{l-1}-m_{l-1}^{i}\right) \lambda_{l-1}+\left(m_{l}-m_{l}^{i}\right) \lambda_{l},
\end{aligned}
$$

where by $m_{l-1}^{i}$ we mean $m_{l-1}$ if $l-i$ is odd and $m_{l}$ if $l-i$ is even, and similarly for $m_{l}^{i}$. These belong to $\Lambda^{+}$when $l-i$ is odd, or when $m_{l-1}=m_{l}$, settling the case of $l$ even. For $l$ odd we have

$$
\begin{gathered}
\bar{\lambda}+w_{i} w_{i+1} \cdots w_{l-1}(\lambda)= \\
2 \sum_{k=1}^{i-2} m_{k} \lambda_{k}+\left(m_{l-1}+m_{l}+2 \sum_{k=i-1}^{l-2} m_{k}\right) \lambda_{i-1} \\
+\left(m_{l}-m_{l-1}^{i}\right) \lambda_{l-1}+\left(m_{l-1}-m_{l}^{i}\right) \lambda_{l} .
\end{gathered}
$$

These belong to $\Lambda^{+}$when $l-i$ is even, or when $m_{l-1}=m_{l}$.

The preceding lemma shows in some sense that the decomposition of the tensor square of an "arbitrary" representation will depend on the rank of the group. Perhaps surprisingly, there are certain representations (whose highest weights can be thought of as rank-independent) whose tensor decompositions are rank-independent, as the following result shows.

(3.5) Proposition. Suppose $\Phi$ is of type $A_{l}, B_{l}, C_{l}$, or $D_{l}$. Then the decomposition of $\lambda_{1}^{m}$ depends only on $m$ whenever $l>m+1$.

Proof. Let $\lambda=\sum_{k=1}^{l} m_{k} \lambda_{k} \in \Lambda^{+}$and suppose that $m_{k}=0$ for $m<k \leq l$, where $m<l-2$. We compute the decomposition of $\lambda_{1} \otimes \lambda$ and see that the component weights are the same regardless of $l$; the claimed result then follows by induction.

Suppose first that $\Phi$ is of type $A_{l}$. Since $\lambda_{1}$ is minimal, $\Pi\left(\lambda_{1}\right)=\mathscr{W} \lambda_{1}[3$, VIII, 7.3]. Keeping the notation used in the proof of (3.4), we have

$$
w_{1 j}\left(\lambda_{1}\right)=-\lambda_{j}+\lambda_{j+1} \text {. }
$$

Since $d\left(\lambda_{1}\right)=\left|\Pi\left(\lambda_{1}\right)\right|=l+1$, we see that

$$
\Pi\left(\lambda_{1}\right)=\left\{-\lambda_{j}+\lambda_{j+1}: 0 \leq j \leq l\right\} .
$$


Because $\left\langle-\lambda_{j}+\lambda_{j+1}+\lambda+\delta, \alpha_{k}\right\rangle \geq 0,0 \leq j \leq l, 1 \leq k \leq l$, there is no cancellation in the Brauer-Klimyk formula, and the tilde function is trivial. Hence the components of $\lambda_{1} \otimes \lambda$ are precisely those $\lambda-\lambda_{j}+\lambda_{j+1}$ which belong to $\Lambda^{+}$(and each occurs with multiplicity 1). Since $m_{j}=0$ for $j>m$, the only possible components are $\lambda-\lambda_{j}+\lambda_{j+1}$ with $0 \leq j \leq m$, and in particular the decomposition is independent of $l$.

We suppose next that $\Phi$ is of type $C_{l}$; again $\lambda_{1}$ is minimal and we find that

$$
\Pi\left(\lambda_{1}\right)=\left\{ \pm\left(\lambda_{j-1}-\lambda_{j}\right): 1 \leq j \leq l\right\}
$$

As before, the components of $\lambda_{1} \otimes \lambda$ are those $\lambda \pm\left(\lambda_{j}-\lambda_{j+1}\right) \in \Lambda^{+}$with $0 \leq j \leq m$, so the decomposition is independent of $l$.

Suppose now that $\Phi$ is of type $D_{l}$; again $\lambda_{1}$ is minimal and we find that

$$
\Pi\left(\lambda_{1}\right)=\left\{ \pm\left(\lambda_{j-1}-\lambda_{j}\right): 1 \leq j \leq l-1\right\} \cup\left\{ \pm\left(\lambda_{l-2}-\lambda_{l-1}-\lambda_{l}\right)\right\} .
$$

As before, and remembering that $m<l-2$, the components of $\lambda_{1} \otimes \lambda$ are those $\lambda \pm\left(\lambda_{j}-\lambda_{j+1}\right) \in \Lambda^{+}$with $0 \leq j \leq m$, so the decomposition is again independent of $l$.

Finally, suppose $\Phi$ is of type $B_{l}$. Here $\lambda_{1}$ is not minimal. We have

$$
\mathscr{W} \lambda_{1}=\left\{ \pm\left(\lambda_{j-1}-\lambda_{j}\right): 1 \leq j \leq l-1\right\} \cup\left\{ \pm\left(\lambda_{l-1}-2 \lambda_{l}\right)\right\} ;
$$

since $d\left(\lambda_{1}\right)=2 l+1$, it follows that there is one weight (also of multiplicity 1) in $\Pi\left(\lambda_{1}\right) \backslash \mathscr{W} \lambda_{1}$. This remaining weight is 0 , since the facts that $\Pi\left(\lambda_{1}\right)$ is saturated and $\left\langle\lambda_{1}, \beta_{1}\right\rangle=2$ together imply that $\lambda_{1}-\beta_{1}=0 \in \Pi\left(\lambda_{1}\right)$. Thus

$$
\Pi\left(\lambda_{1}\right)=\left\{ \pm\left(\lambda_{j-1}-\lambda_{j}\right): 1 \leq j \leq l-1\right\} \cup\left\{0, \pm\left(\lambda_{l-1}-2 \lambda_{l}\right)\right\} .
$$

Since $m<l-2,\left\langle\lambda-\lambda_{l-1}+2 \lambda_{l}+\delta, \alpha_{l-1}\right\rangle=0$ so that $\operatorname{sgn}\left(\lambda-\lambda_{l-1}+2 \lambda_{l}+\delta\right)=0$ and the weight $-\lambda_{l-1}+2 \lambda_{l}$ contributes nothing to the sum in (3.1). Observe that $w_{l}\left(\lambda+\lambda_{l-1}-2 \lambda_{l}+\delta\right)=\lambda+\delta$, so that $\operatorname{sgn}\left(\lambda+\lambda_{l-1}-2 \lambda_{l}+\delta\right)=-1$ and $\left(\lambda+\lambda_{l-1}-2 \lambda_{l}+\delta\right)^{\sim}-\delta=\lambda$. Thus the contributions from the weights 0 and $\lambda_{l-1}-2 \lambda_{l}$ cancel, and the decomposition of $\lambda_{1} \otimes \lambda$ is again those $\lambda \pm\left(\lambda_{j}-\lambda_{j+1}\right) \in$ $\Lambda^{+}$with $0 \leq j \leq m$.

(3.6) Corollary. Suppose $\Phi$ is of type $A_{l}$. Then the decomposition of $\left(\overline{\lambda_{1}}\right)^{m}$ depends only on $m$ whenever $l>m+1$. Furthermore, the decomposition of $\lambda_{1}^{m} \otimes\left(\overline{\lambda_{1}}\right)^{n}$ depends only on $m$ and $n$ whenever $l>m+n$.

Proof. The first assertion follows from (3.5) via the symmetry of $\lambda_{l}=\bar{\lambda}_{1}$. The second follows from similar calculations to those in (3.5): if $\lambda=\sum_{k=1}^{l} m_{k} \lambda_{k} \in$ $\Lambda^{+}$has $m_{k}=0$ for $m<k \leq l-n$ then the components of $\lambda_{1} \otimes \lambda$ are those $\lambda-\lambda_{j}+\lambda_{j+1}$ and $\lambda+\overline{\left(\lambda_{j}-\lambda_{j+1}\right)}$ which lie in $\Lambda^{+}$, where $0 \leq j \leq \max (m, n)$.

(3.7) Conjecture. Let $\Phi$ be of type $A_{l}, B_{l}, C_{l}$, or $D_{l}$ and suppose $\mu \leq \lambda_{1}^{m}$ and $\nu \leq \lambda_{1}^{n}$. Provided $l>m+n+1$ we know that the decomposition of $\lambda_{1}^{m+n}$ is independent of $l$, and hence the possible decompositions of $\mu \otimes \nu$ are finite in number. We conjecture that in fact $\mu \otimes \nu$ has the same decomposition regardless of $l$, provided that $l>m+n+1$.

\section{4. $m$-FOLD FTR SETS AND CONSTRUCTIONS OF (CENTRAL) $\Lambda(p)$ SETS}

We define a special class of sets closely related to the FTR sets of [5]. Because our present interest is with connected compact groups, we make use of the 
following structure theorem in our definition. A definition for arbitrary compact groups (along lines similar to the definition of FTR sets in [18]) is possible, but unnecessarily awkward for our purposes.

(4.1) Theorem. Let $G$ be a compact connected group. Then there exist a group of the form $\mathscr{G}=T \times \prod_{\alpha \in A} G_{\alpha}$, where $T$ is the connected component of the identity of the centre of $G$ and $\left(G_{\alpha}\right)_{\alpha \in A}$ is a family of almost simple simplyconnected compact Lie groups, and an epimorphism $\pi: \mathscr{G} \rightarrow G$ whose kernel is a totally disconnected closed subgroup of the centre of $\mathscr{G}$ with the property that $(\operatorname{ker} \pi) \cap T$ contains only the identity.

Further, if $G$ is tall, then $T$ is trivial, and for each $l \in \mathbb{N}$ there are only finitely many $G_{\alpha}$ having rank $l$.

Proof. This is taken from $[15,6.5 .6]$ and $[5,6.3]$.

Definition. Let $G$ be a compact connected group with covering group $\mathscr{G}$ and epimorphism $\pi: \mathscr{G} \rightarrow G$ as in (4.1). Let $F=\operatorname{FTR}(\mathscr{G})$ be the FTR set of $\mathscr{G}$ (in the sense of [18]). The $m$-fold FTR set of $G$ is then

$$
\operatorname{FTR}^{m}(G)=\left\{\sigma \in \widehat{G}: \sigma \circ \pi \in F^{m}\right\} .
$$

Let $P$ be any subset of $F$ having the property that (i) $P \cup \bar{P} \subseteq F$ and (ii) $P \cap \bar{P}=\varnothing$. Then we say that $\left\{\sigma \in \widehat{G}: \sigma \circ \pi \in P^{m}\right\}$ is a partial m-fold FTR set of $G$.

Note that a 1 -fold FTR set is just an FTR set, and that $\mathrm{FTR}^{m}(G)$ is quite a different creature from $\operatorname{FTR}_{m}(G)$, the FTR set of order $m$ defined in [18]. Also note that in general $\operatorname{FTR}^{m}(G) \neq(\operatorname{FTR}(G))^{m}$ : consider $G=\prod_{n \geq 2} P S O(2 n)$, when $\operatorname{FTR}(G)$ is empty, but $\operatorname{FTR}^{2}(G) \supseteq\left\{\lambda_{2} \circ \pi_{n}: n \geq 2\right\} \quad\left(\pi_{n}: G \rightarrow P S O(2 n)\right.$ denotes the canonical projection). Since $1 \in \operatorname{FTR}^{2}(G)$ whenever $\operatorname{FTR}(\mathscr{G}) \neq \varnothing$, it follows that $\operatorname{FTR}^{m}(G) \subseteq \operatorname{FTR}^{m+2}(G)$.

When $\mathscr{G}=\prod_{\alpha \in A} G_{\alpha}$, with the $G_{\alpha}$ simply-connected compact almost simple Lie groups (and, for convenience, other than $\operatorname{Spin}(8)$ or $\operatorname{Spin}(5)$ ) the elements of $\operatorname{FTR}^{m}(\mathscr{G})$ are easily described. For each $\sigma=\left(\sigma_{\alpha}\right)_{\alpha \in A} \in \mathrm{FTR}^{m}(\mathscr{G})$ there is a family $\left(\left(i_{\alpha}, j_{\alpha}\right)\right)_{\alpha \in A}$ of nonnegative exponents such that $\sum_{\alpha \in A}\left(i_{\alpha}+j_{\alpha}\right)=m$ and for each $\alpha \in A, \sigma_{\alpha} \leq \varsigma_{\alpha}^{i_{\alpha}} \otimes{\overline{\zeta_{\alpha}}}^{j_{\alpha}}$. For each $\sigma \in P$, a partial $m$-fold FTR set for $\mathscr{G}$, there is a family $\left(i_{\alpha}\right)_{\alpha \in A}$ such that $\sum_{\alpha \in A} i_{\alpha}=m$, where for each $\alpha \in A^{\prime}=\left\{\alpha \in A: G_{\alpha}=S U\left(n_{\alpha}\right)\right\}$ either $\sigma_{\alpha} \leq \varsigma_{\alpha}^{i_{\alpha}}$ or $\sigma_{\alpha} \leq{\overline{\zeta_{\alpha}}}^{i_{\alpha}}$, and $\sigma_{\alpha}=1$ for $\alpha \in A \backslash A^{\prime}$.

An obvious corollary of Proposition (3.5) is that for $G$ a compact connected almost simple Lie group, the number of irreducible subrepresentations of $\varsigma^{j} \otimes \bar{\zeta}^{k}$ is bounded by a constant depending only on $m$ whenever $0 \leq j, k \leq m$. Thus in this case a bound for the cardinality of $\operatorname{FTR}^{m}(G)$ depends only on $m$. This property is also a consequence of the fact that the Sidon constant of $\operatorname{FTR}(G)$, and hence also $\eta_{p}(\operatorname{FTR}(G))$, is independent of $G[5,4.5 .2 ; 18,3.2]$.

We will first prove that $m$-fold FTR sets are central $\Lambda(p)$ sets for all $p \in$ $(2, \infty)$. As every (central) $\Lambda(p)$ set is (central) $\Lambda(q)$ for all $q<p$ it suffices to show that these sets are central $\Lambda(2 s)$ sets for all positive integers $s$. Since $m$-fold FTR sets are defined via a covering group, we first require information about how $\Lambda(p)$ sets are preserved by group homomorphisms. 
(4.2) Proposition. Let $G, H$ be compact groups and suppose there is an epimorphism $\pi: G \rightarrow H$. Then for every $E \subseteq \widehat{H}$ we have

(i) $\eta_{p}(E)=\eta_{p}(E \circ \pi)$,

(ii) $\zeta_{p}(E)=\zeta_{p}(E \circ \pi)$,

(iii) $\eta_{p}^{0}(E)=\eta_{p}^{0}(E \circ \pi)$, and

(iv) $\zeta_{p}^{0}(E)=\zeta_{p}^{0}(E \circ \pi)$.

Proof. Parts (iii) and (iv) are easy corollaries of (i) and (ii), and the proof of (ii) is obtained from that of (i) by replacing $\mathscr{T}$ with $\mathscr{T}^{z}$ throughout.

Let $f \in \mathscr{T}_{E}(H)$; then $f \circ \pi \in \mathscr{T}_{E \circ \pi}(G)$, and for any $1 \leq q<\infty$

$$
\begin{aligned}
\|f \circ \pi\|_{q}^{q} & =\int_{G}|f \circ \pi(g)|^{q} d g \\
& =\int_{G / \operatorname{ker} \pi} \int_{\operatorname{ker} \pi}|f \circ \pi(x g)|^{q} d g d \mathbf{x} \\
& (\text { where }\{x\} \text { is a transversal to ker } \pi \text { in } G) \\
& =\int_{G / \operatorname{ker} \pi}|f(\mathbf{x})|^{q} \int_{\operatorname{ker} \pi} d g d \mathbf{x} \\
& (\text { since } f \circ \pi \text { is constant on ker } \pi \text {-cosets) } \\
& =\int_{H}|f(h)|^{q} d h \quad(\text { since } G / \text { ker } \pi \cong H) \\
& =\|f\|_{q}^{q} .
\end{aligned}
$$

For the converse, observe that every $\phi \in \mathscr{T}_{E \circ \pi}$ has the form $f \circ \pi$ for some $f \in \mathscr{T}_{E}(H)$.

The proof of the next result has a strong abelian tang (cf. [14, 5.13]).

(4.3) Proposition. Let $\mathscr{G}=\prod_{\alpha \in A} G_{\alpha}$ be a product of connected almost simple compact Lie groups, and suppose the ranks of the $G_{\alpha}$ are bounded. Then for any $m, \operatorname{FTR}^{m}(\mathscr{G})$ is a $\Lambda(p)$ set for all $p \in(2, \infty)$.

Proof. In view of (4.2) and the definition of $\mathrm{FTR}^{m}(\mathscr{G})$ we may suppose that the $G_{\alpha}$ are simply connected.

Write $P_{\alpha}$ for a minimal subset of $\widehat{G}_{\alpha}$ having the property that

$$
P_{\alpha} \cup \overline{P_{\alpha}} \cup\{1\}=\left\{\sigma \in \widehat{G}_{\alpha}: \sigma \leq \varsigma_{\alpha}^{j} \otimes{\overline{\zeta_{\alpha}}}^{k}, j, k \leq m\right\} ;
$$

since the ranks of the $G_{\alpha}$ are bounded, it follows that $\left|P_{\alpha}\right| \leq N(m)$. For $1 \leq k \leq m$ let

$$
E_{k}=\left\{\left(\sigma_{\alpha}\right)_{\alpha \in A} \in \hat{\mathscr{G}}: \sigma_{\alpha} \in P_{\alpha} \cup \overline{P_{\alpha}} \cup\{1\} \text { and } \sigma_{\alpha} \neq 1 \text { for exactly } k \alpha \text { 's }\right\} \text {. }
$$

Since $\operatorname{FTR}^{m}(\mathscr{G}) \subseteq\{1\} \cup \bigcup_{k=1}^{m} E_{k}$, and since for $p>2$ a finite union of $\Lambda(p)$ sets is again $\Lambda(p)$, it suffices to show that each $E_{k}$ is a $\Lambda(p)$ set.

Let $X=\prod_{\alpha \in A} \prod_{\sigma \in P_{\alpha}} U(d(\sigma))$ and denote by $\varsigma_{\alpha, \sigma}$ the self-representation of the corresponding factor. Put $C=\sup \left\{2\left|P_{\alpha}\right| d(\sigma)^{2}: \sigma \in P_{\alpha}, \alpha \in A\right\} ; C$ is 
finite since the $G_{\alpha}$ have bounded rank. For $x=\left(x_{\alpha, \sigma}\right)_{\alpha \in A, \sigma \in P_{\alpha}} \in X, g=$ $\left(g_{\alpha}\right)_{\alpha \in A} \in G$, and a finite set $B \subseteq A$ let

$$
Q_{B}(x, g)=\prod_{\alpha \in B}\left(1+\frac{1}{C} \sum_{\sigma \in P_{\alpha}} d(\sigma) \operatorname{tr}\left(\varsigma_{\alpha, \sigma}\left(x_{\alpha, \sigma}\right) \sigma\left(g_{\alpha}\right)+\overline{\varsigma_{\alpha, \sigma}\left(x_{\alpha, \sigma}\right) \sigma\left(g_{\alpha}\right)}\right)\right)
$$

and let $Q_{B, x}(g)=Q_{B}(x, g)$. The choice of $C$ ensures that $Q_{B, x} \geq 0$. We have

$$
\begin{aligned}
Q_{B, x}(g)=1+\sum_{k=1}^{|B|} \frac{1}{C^{k}} \sum_{\substack{\Gamma \subseteq B,|\Gamma|=k \\
\varepsilon_{1}, \ldots, \varepsilon_{k}= \pm 1}} \sum_{\sigma_{j} \in P_{\alpha_{j}}} d\left(\sigma_{1}\right) \cdots d\left(\sigma_{k}\right) \\
\cdot \operatorname{tr}\left(\left(\varsigma_{\alpha_{1}, \sigma_{1}}^{\varepsilon_{1}} \times \cdots \times \varsigma_{\alpha_{k}, \sigma_{k}}^{\varepsilon_{k}} \times 1\right)(x)\left(\sigma_{1}^{\varepsilon_{1}} \times \cdots \times \sigma_{k}^{\varepsilon_{k}} \times 1\right)(g)\right),
\end{aligned}
$$

where $\alpha_{1}=\alpha_{1}(\Gamma), \ldots, \alpha_{k}=\alpha_{k}(\Gamma)$ denote the elements of $\Gamma$ in some order; since each $\sigma_{1}^{\varepsilon_{1}} \times \cdots \times \sigma_{k}^{\varepsilon_{k}} \times 1$ is nontrivial and irreducible, it follows that $\left\|Q_{B, x}\right\|_{1}=1$.

Given any $f \in \mathscr{T}(G)$ we have

$$
\begin{aligned}
& \left(Q_{B, x} * f\right)(g)=\widehat{f}(1)+\sum_{k=1}^{|B|} \frac{1}{C^{k}} \sum_{\substack{\Gamma \subseteq B,|\Gamma|=k \\
\varepsilon_{1}, \ldots, \varepsilon_{k}= \pm 1}} \sum_{\sigma_{j} \in P_{\alpha_{j}}} d\left(\sigma_{1}\right) \cdots d\left(\sigma_{k}\right) \\
& \cdot \operatorname{tr}\left(\left(\varsigma_{\alpha_{1}, \sigma_{1}}^{\varepsilon_{1}} \times \cdots \times \varsigma_{\alpha_{k}, \sigma_{k}}^{\varepsilon_{k}} \times 1\right)(x)\left(\sigma_{1}^{\varepsilon_{1}} \times \cdots \times \sigma_{k}^{\varepsilon_{k}} \times 1\right)(g) \widehat{f}\left(\sigma_{1}^{\varepsilon_{1}} \times \cdots \times \sigma_{k}^{\varepsilon_{k}} \times 1\right)\right) ;
\end{aligned}
$$

let $F_{B, g}(x)=\left(Q_{B, x} * f\right)(g)$ and observe that $F_{B, g} \in \mathscr{T}(X)$, with

$$
\begin{aligned}
\widehat{F_{B, g}} & \left(\varsigma_{\alpha_{1}, \sigma_{1}}^{\varepsilon_{1}} \times \cdots \times \varsigma_{\alpha_{k}, \sigma_{k}}^{\varepsilon_{k}} \times 1\right) \\
& =\frac{1}{C^{k}}\left(\sigma_{1}^{\varepsilon_{1}} \times \cdots \times \sigma_{k}^{\varepsilon_{k}} \times 1\right)(g) \widehat{f}\left(\sigma_{1}^{\varepsilon_{1}} \times \cdots \times \sigma_{k}^{\varepsilon_{k}} \times 1\right) .
\end{aligned}
$$

In [10, Theorem 2.2] it is shown that

$$
\begin{aligned}
D_{k}=\left\{\left(\tau_{\alpha, \sigma}\right)_{\alpha \in A, \sigma \in P_{\alpha}} \in \widehat{X}:\right. & \text { exactly } k \text { of the } \tau_{\alpha, \sigma} \\
& \text { are nontrivial, when } \left.\tau_{\alpha, \sigma}=\varsigma_{\alpha, \sigma}^{ \pm 1}\right\}
\end{aligned}
$$

is a $\Lambda(p)$ set for every $p \in(2, \infty)$. Since

$$
\operatorname{tr}\left|\left(\sigma_{1}^{\varepsilon_{1}} \times \cdots \times \sigma_{k}^{\varepsilon_{k}} \times 1\right)(g) \widehat{f}\left(\sigma_{1}^{\varepsilon_{1}} \times \cdots \times \sigma_{k}^{\varepsilon_{k}} \times 1\right)\right|^{2}=\operatorname{tr}\left|\widehat{f}\left(\sigma_{1}^{\varepsilon_{1}} \times \cdots \times \sigma_{k}^{\varepsilon_{k}} \times 1\right)\right|^{2}
$$

it follows from $(*)$ that for sufficiently large $B$,

$$
\left\|\left.\widehat{F_{B, g}}\right|_{D_{k}}\right\|_{2}^{2} \geq \frac{1}{C^{2 k}}\left\|\left.\widehat{f}\right|_{E_{k}}\right\|_{2}^{2}
$$

the inequality arising because $P_{\alpha} \cap \overline{P_{\alpha}}$ need not be empty. Thus

$$
\left\|\left.\widehat{f}\right|_{E_{k}}\right\|_{2} \leq C^{k} \eta_{p}\left(D_{k}\right)\left\|F_{B, g}\right\|_{q}
$$

by the dual characterization of $\Lambda(p)$ sets [9, 37.9], where $\frac{1}{p}+\frac{1}{q}=1$.

Finally, observe that $\left\|Q_{B, x} * f\right\|_{q} \leq\left\|Q_{B}, x\right\|_{1}\|f\|_{q}=\|f\|_{q}$ so that

$$
\|f\|_{q}^{q} \geq \int_{X}\left\|Q_{B, x} * f\right\|_{q}^{q} d x=\int_{G}\left\|F_{B, g}\right\|_{q}^{q} d g
$$


hence for some $g \in G,\left\|F_{B, g}\right\|_{q} \leq\|f\|_{q}$ and $E_{k}$ is indeed $\Lambda(p)$ (with $\left.\eta_{p}\left(E_{k}\right) \leq C^{k} \eta_{p}\left(D_{k}\right)\right)$.

(4.4) Theorem. Let $G$ be a connected compact group. Then $\operatorname{FTR}^{m}(G)$ is a central $\Lambda(p)$ set for all $p \in(2, \infty)$.

Proof. The case $m=1$ holds because FTR $(G)$ is Sidon [18, 3.4]. So let $s \geq 2$ and $m \geq 2$ be given. Because of the definition of $\operatorname{FTR}^{m}(G)$ together with (4.2) it suffices to consider $G=\prod_{\alpha \in A} G_{\alpha}$, where the $G_{\alpha}$ are as in the structure theorem, Theorem (4.1). Indeed, since a set is (central) $\Lambda(p)$ if and only if every countable subset is (central) $\Lambda(p)$ we may suppose without loss of generality that $A=\mathbb{N}$, that is, $G=\prod_{i \geq 1} G_{i}$.

We will use Benke's characterization [1, $\S 2]$ of central $\Lambda(2 s)$ sets: $E \subseteq \widehat{G}$ is a central $\Lambda(2 s)$ set if and only if there is a constant $C$ such that every sequence $\left\{a_{j}\right\}_{j \geq 1}$ of positive numbers satisfies

$$
\sum_{\sigma \in \widehat{G}}\left(\sum_{\sigma_{j_{1}}, \ldots, \sigma_{j_{s}} \in E} a_{j_{1}} \cdots a_{j_{s}}\left[\sigma: \sigma_{j_{1}} \otimes \cdots \otimes \sigma_{j_{s}}\right]\right)^{2} \leq C\left(\sum_{j \geq 1} a_{j}^{2}\right)^{s}
$$

where $[\sigma: \gamma]$ denotes the multiplicity of $\sigma$ in $\gamma$.

Consider $\sigma_{j_{1}}, \ldots, \sigma_{j_{s}} \in E=\mathrm{FTR}^{m}(G)$ : write ${ }_{k} \sigma_{i}$ for the projection of $\sigma_{j_{k}}$ on $\widehat{G}_{i}$. The decomposition of $\sigma_{j_{1}} \otimes \cdots \otimes \sigma_{j_{s}}$ is determined completely by the decompositions (only finitely many of which are nontrivial) of ${ }_{1} \sigma_{i} \otimes \cdots \otimes{ }_{s} \sigma_{i}$ on $G_{i}$. Write $A^{\prime}=\left\{i: G_{i}\right.$ is of type $A, B, C$ or $D$ and has rank $\left.\geq m s+2\right\}$ and consider $i \in A^{\prime}$ (note that $m s+2 \geq 6$ so that $G_{i}$ is not one of the troublesome groups $\operatorname{Spin}(8)$ or $\operatorname{Spin}(5))$. Then ${ }_{1} \sigma_{i} \otimes \cdots \otimes{ }_{s} \sigma_{i}$ is a subrepresentation of $\varsigma_{i}^{p} \otimes \bar{\zeta}_{i}^{q}$ for some $p, q$ with $0 \leq p+q \leq m s$. By (3.5), the decomposition of $\varsigma_{i}^{p} \otimes{\overline{S_{i}}}^{q}$ is independent of the rank of $G_{i}$. There are thus only a finite number of possible decompositions for ${ }_{1} \sigma_{i} \otimes \cdots \otimes{ }_{s} \sigma_{i}$, and further, there are only finitely many distinct (in terms of labelling by highest weights) ${ }_{1} \sigma_{i} \otimes \cdots \otimes{ }_{s} \sigma_{i}$ which can arise. It follows that there is a finite collection $\mathbb{G}(m, s)$ of simplyconnected almost simple compact Lie groups with the property that for each $i \in A^{\prime}$ we can find $G_{i}^{\prime} \in \mathbb{G}$ having the same Lie type as $G_{i}$ so that, writing $\sigma_{i}^{\prime}$ for the representation of $G_{i}^{\prime}$ which has the "same" highest weight as $\sigma_{i}$, all decompositions of ${ }_{1} \sigma_{i} \otimes \cdots \otimes{ }_{s} \sigma_{i}$ which arise as the $\sigma_{k}$ range over $\operatorname{FTR}^{m}(G)$ coincide with the decompositions of ${ }_{1} \sigma_{i}^{\prime} \otimes \cdots \otimes{ }_{s} \sigma_{i}^{\prime}$. (Note that Conjecture (3.7) implies that $\mathbb{G}(m, s)$ contains exactly four groups: the simply-connected groups of types $A_{m s+2}, B_{m s+2}, C_{m s+2}$ and $D_{m s+2}$.) For $i \notin A^{\prime}$, put $G_{i}^{\prime}=G_{i}$, let $G^{\prime}=\prod_{i \geq 1} G_{i}^{\prime}$, and observe that $\sup _{i \geq 1}\left\{\right.$ rank $\left.G_{i}^{\prime}\right\}<\infty$.

It is now clear that the left-hand side of $(*)$ is equal to

$$
\sum_{\sigma \in \widehat{G}^{\prime}}\left(\sum_{\sigma_{j_{1}}, \ldots, \sigma_{j_{s}} \in E^{\prime}} a_{j_{1}} \cdots a_{j_{s}}\left[\sigma: \sigma_{j_{1}} \otimes \cdots \otimes \sigma_{j_{s}}\right]\right)^{2}
$$

where $E^{\prime}=\mathrm{FTR}^{m}\left(G^{\prime}\right)$. But since $E^{\prime}$ is central $\Lambda(2 s)$ for all $s>1$ by Proposition (4.3), it follows that $E=\operatorname{FTR}^{m}(G)$ is also.

Rider [16, Theorem 5] has shown that a set of irreducible representations is a $\Lambda(2 s)$ set if and only if it is both local $\Lambda(2 s)$ and central $\Lambda(2 s)$. Our next 
goal is to demonstrate that certain partial $m$-fold FTR sets are local $\Lambda(p)$ sets; we do this by extending ideas of [8].

(4.5) Lemma. Let $G$ be either $U(n)$ or $S U(n)$ and let $x=\left(x_{i j}\right) \in G$. Then for every positive integer $s$ there is a constant $B(s)$ such that

$$
\int_{G}\left|x_{i j}\right|^{2 s} d x \leq \frac{B(s)}{n^{s}} .
$$

This result is essentially proved in [8, Lemma 1] for $U(n)$; the same proof works for $S U(n)$.

(4.6) Lemma. Let $G$ be either $U(n)$ or $S U(n)$ and let $n>2 s$. Then

$$
\int_{G} x_{i_{1} j_{1}} \overline{x_{i_{1}^{\prime} j_{1}^{\prime}}} \cdots x_{i_{s} j_{s}} \overline{x_{i_{s}^{\prime} j_{s}^{\prime}}} d x=0
$$

unless $\left\{i_{1}^{\prime}, \ldots, i_{s}^{\prime}\right\}$ is a rearrangement of $\left\{i_{1}, \ldots, i_{s}\right\}$ and $\left\{j_{1}^{\prime}, \ldots, j_{s}^{\prime}\right\}$ is a rearrangement of $\left\{j_{1}, \ldots, j_{s}\right\}$.

Proof. Consider a diagonal matrix $w \in S U(n)$ : left invariance of the Haar integral ensures that

$$
\begin{aligned}
\int_{G} x_{i_{1} j_{1}} \overline{x_{i_{1}^{\prime} j_{1}^{\prime}}} \cdots x_{i_{s} j_{s}} \overline{x_{i_{s}^{\prime} j_{s}^{\prime}}} d x & =\int_{G}(w x)_{i_{1} j_{1}} \overline{(w x)_{i_{1}^{\prime} j_{1}^{\prime}}} \cdots(w x)_{i_{s} j_{s}} \overline{(w x)_{i_{s}^{\prime} j_{s}^{\prime}}} d x \\
& =w_{i_{1} i_{1}} \overline{w_{i_{1}^{\prime} i_{1}^{\prime}}} \cdots w_{i_{s} i_{s}} \overline{w_{i_{s}^{\prime} i_{s}^{\prime}}} \int_{G} x_{i_{1} j_{1}} \overline{i_{i_{1}^{\prime} j_{1}^{\prime}}} \cdots x_{i_{s} j_{s}} \overline{x_{s_{s}^{\prime} j_{s}^{\prime}}} d x .
\end{aligned}
$$

Since $n>2 s$ we can choose $w_{k k}$ to be an arbitrary complex number of modulus 1 for each $k \in\left\{i_{1}, \ldots, i_{s}\right\} \cup\left\{i_{1}^{\prime}, \ldots, i_{s}^{\prime}\right\}$, and so if the integral is nonzero then $\left\{i_{1}^{\prime}, \ldots, i_{s}^{\prime}\right\}$ must be a rearrangement of $\left\{i_{1}, \ldots, i_{s}\right\}$.

A similar argument using right invariance of the Haar integral completes the proof.

(4.7) Theorem. Let $s$ and $m$ be positive integers and let $\mathscr{G}=\prod_{\alpha \in A} G_{\alpha}$ where each $G_{\alpha}$ is either $U\left(n_{\alpha}\right)$ or $S U\left(n_{\alpha}\right)$ and $n_{\alpha}>2 m s$. Write $\varsigma_{\alpha}$ for the selfrepresentation of $G_{\alpha}$ and put

$$
P_{m}=\left\{\left(\gamma_{\alpha}\right)_{\alpha \in A}: \text { at most } m \text { of the } \gamma_{\alpha}\right. \text { are nontrivial, }
$$

$$
\text { when } \left.\gamma_{\alpha}=\varsigma_{\alpha}^{ \pm j_{n}} \text {, some } 1 \leq j_{\alpha} \leq m\right\} \text {. }
$$

Then there is a constant $C(s, m)$ so that for any $\gamma \in P_{m}$ and $A$ any $d(\gamma) \times d(\gamma)$ matrix,

$$
\int_{\mathscr{G}}|\operatorname{tr} A \gamma|^{2 s} \leq \frac{C(s, m)}{d(\gamma)^{s}}\left(\operatorname{tr} A A^{*}\right)^{s} .
$$

Proof. The case $m=1$ and $\mathscr{G}=U(n)$ is proved in [8, Lemma 1]. We will only present the proof for the special cases

$$
\int_{G_{1} \times G_{2}}\left|\operatorname{tr} A\left(\varsigma_{1} \times \varsigma_{2}\right)(x, y)\right|^{2 s} d x d y
$$

and

$$
\int_{G}|\operatorname{tr} A(\varsigma \otimes \varsigma)(x)|^{2 s} d x ;
$$

it should be clear that our techniques generalize. 
In what follows, $C=C(s, 2)$ will denote a constant, whose value may change from line to line. We use $b_{i j k l}$ to denote the entry in the $(i, j) \otimes(k, l)$ position of the $n m \times n m$ matrix $B$; the diagonal entries are $b_{j j k k}$ so that

$$
\operatorname{tr} A\left(\varsigma_{1} \times \varsigma_{2}\right)(x, y)=\sum_{j, k}\left(A\left(\varsigma_{1}(x) \otimes \varsigma_{2}(y)\right)\right)_{j j k k}=\sum_{i, j, k, l} a_{j i k l} x_{i j} y_{l k},
$$

and

$$
\operatorname{tr} A(\varsigma \otimes \varsigma)(x)=\sum_{i, j, k, l} a_{j i k l} x_{i j} x_{l k}
$$

Hence

$$
\begin{aligned}
\int_{G_{1} \times G_{2}} \mid & \left.\operatorname{tr} A\left(s_{1} \times \varsigma_{2}\right)(x, y)\right|^{2 s} d x d y \\
= & \sum_{\substack{i_{1}, \ldots, i_{s} ; i_{1}^{\prime}, \ldots, i_{s}^{\prime} \\
j_{1}, \ldots, j_{s} ; j_{1}^{\prime}, \ldots, j_{s}^{\prime} \\
k_{1}, \ldots, k_{s} ; l_{1}^{\prime}, \ldots, k_{s}^{\prime} \\
l_{1}, \ldots, l_{s} ; l_{1}^{\prime}, \ldots, l_{s}^{\prime}}} a_{j_{1} i_{1} k_{1} l_{1}} \overline{a_{j_{1}^{\prime} i_{1}^{\prime} k_{1}^{\prime} l_{1}^{\prime}}} \cdots a_{j_{s} i_{s} k_{s} l_{s}} \overline{a_{j_{s}^{\prime} i_{s}^{\prime} k_{s}^{\prime} l_{s}^{\prime}}} \\
& \cdot \int_{G_{1}} x_{i_{1} j_{1}} \overline{x_{i_{1}^{\prime} j_{1}^{\prime}}} \ldots x_{i_{s} j_{s}} \overline{x_{i_{s}^{\prime} j_{s}^{\prime}}} d x \int_{G_{2}} y_{l_{1} k_{1}} \overline{y_{l_{1}^{\prime} k_{1}^{\prime}}} \ldots y_{l_{s} k_{s}} \overline{y_{l_{s}^{\prime} k_{s}^{\prime}}} d y .
\end{aligned}
$$

According to Lemma (4.6) the above integrals are zero unless $i_{1}^{\prime}, \ldots, i_{s}^{\prime}$ is a rearrangement of $i_{1}, \ldots, i_{s} ; j_{1}^{\prime}, \ldots, j_{s}^{\prime}$ is a rearrangement of $j_{1}, \ldots, j_{s}$; $k_{1}^{\prime}, \ldots, k_{s}^{\prime}$ is a rearrangement of $k_{1}, \ldots, k_{s}$; and $l_{1}^{\prime}, \ldots, l_{s}^{\prime}$ is a rearrangement of $l_{1}, \ldots, l_{s}$. Thus the integral reduces to

$$
\int_{G_{1}} \int_{G_{2}} \sum\left|\sum a_{j_{1} i_{1} k_{1} l_{1}} \cdots a_{j_{s} i_{s} k_{s} l_{s}} x_{i_{1} j_{1}} \cdots x_{i_{s} j_{s}} y_{l_{1} k_{1}} \cdots y_{l_{s} k_{s}}\right|^{2} d y d x
$$

where the outer sum is over $I_{1} \leq I_{2} \leq \cdots \leq I_{s}, J_{1} \leq J_{2} \leq \cdots \leq J_{s}$, $K_{1} \leq K_{2} \leq \cdots \leq K_{s}$, and $L_{1} \leq L_{2} \leq \cdots \leq L_{s}$, and the inner sum is over $i_{1}, \ldots, i_{s}$ permutations of $\left\{I_{1}, \ldots, I_{s}\right\}, j_{1}, \ldots, j_{s}$ permutations of $\left\{J_{1}, \ldots, J_{s}\right\}, k_{1}, \ldots, k_{s}$ permutations of $\left\{K_{1}, \ldots, K_{s}\right\}$, and $l_{1}, \ldots, l_{s}$ permutations of $\left\{L_{1}, \ldots, L_{s}\right\}$. This expression is dominated by

$$
2(s !)^{4} \sum \sum\left|a_{j_{1} i_{1} k_{1} l_{1}} \cdots a_{j_{s} i_{s} k_{s} l_{s}}\right|^{2} \int_{G_{1}}\left|x_{i_{1} j_{1}} \cdots x_{i_{s} j_{s}}\right|^{2} d x \int_{G_{2}}\left|y_{l_{1} k_{1}} \cdots y_{l_{s} k_{s}}\right|^{2} d y,
$$

the summations being as before. By Hölder's inequality

$$
\int_{G_{1}}\left|x_{i_{1} j_{1}} \cdots x_{i_{s} j_{s}}\right|^{2} d x \leq\left(\int_{G_{1}}\left|x_{i_{1} j_{1}}\right|^{2 s}\right)^{1 / s} \cdots\left(\int_{G_{1}}\left|x_{i_{s} j_{s}}\right|^{2 s}\right)^{1 / s}
$$

so that after applying Lemma (4.5) we find

$$
\begin{aligned}
\int_{G_{1} \times G_{2}}\left|\operatorname{tr} A\left(\varsigma_{1} \times \varsigma_{2}\right)(x, y)\right|^{2 s} d x d y & \leq \frac{C}{d\left(\varsigma_{1} \times \varsigma_{2}\right)^{s}} \sum \sum\left|a_{j_{1} i_{1} k_{1} l_{1}} \cdots a_{j_{s} i_{s} k_{s} l_{s}}\right|^{2} \\
& \leq \frac{C}{d\left(\varsigma_{1} \times \varsigma_{2}\right)^{s}}\left(\operatorname{tr} A A^{*}\right)^{s} .
\end{aligned}
$$




$$
\begin{aligned}
& \text { Similarly } \\
& \int_{G}|\operatorname{tr} A(\varsigma \otimes \varsigma)(x)|^{2 s} d x=\sum_{\begin{array}{c}
i_{1}, \ldots, i_{s} ; i_{1}^{\prime}, \ldots, i_{s}^{\prime} \\
j_{1}, \ldots, j_{s} ; j_{1}^{\prime}, \ldots, j_{s}^{\prime} \\
k_{1}, \ldots, k_{s} ; k_{1}^{\prime}, \ldots, k_{s}^{\prime} \\
l_{1}, \ldots, l_{s} ; l_{1}^{\prime}, \ldots, l_{s}^{\prime}
\end{array}} a_{j_{1} i_{1} k_{1} l_{1}} \overline{a_{j_{1}^{\prime} i_{1}^{\prime} k_{1}^{\prime} l_{1}^{\prime}} \cdots a_{j_{s} i_{s} k_{s} l_{s}} \overline{a_{j_{s}^{\prime} i_{s}^{\prime} k_{s}^{\prime} l_{s}^{\prime}}}} \\
& \quad \int_{G} x_{i_{1} j_{1}} \overline{x_{i_{1}^{\prime} j_{1}^{\prime}}} \cdots x_{i_{s} j_{s}} \overline{x_{i_{s}^{\prime} j_{s}^{\prime}}} x_{l_{1} k_{1}} \overline{x_{l_{1}^{\prime} k_{1}^{\prime}}} \cdots x_{l_{s} k_{s}} \overline{x_{l_{s}^{\prime} k_{s}^{\prime}}} d x,
\end{aligned}
$$

which by Lemma (4.6) simplifies to

$$
\int_{G} \sum\left|\sum a_{j_{1} i_{1} k_{1} l_{1}} \cdots a_{j_{s} i_{s} k_{s} l_{s}} x_{i_{1} j_{1}} \cdots x_{i_{s} j_{s}} x_{l_{1} k_{1}} \cdots x_{l_{s} k_{s}}\right|^{2} d x,
$$

where the outer sum is over $I_{1} \leq I_{2} \leq \cdots \leq I_{2 s}$ and $J_{1} \leq J_{2} \leq \cdots \leq J_{2 s}$, and the inner sum is over $i_{1}, \ldots, i_{s}, l_{1}, \ldots, l_{s}$ permutations of $\left\{I_{1}, \ldots, I_{2 s}\right\}$ and $j_{1}, \ldots, j_{s}, k_{1}, \ldots, k_{s}$ permutations of $\left\{J_{1}, \ldots, J_{2 s}\right\}$. Again via the triangle inequality, Hölder's inequality, and Lemma (4.5) we conclude that

$$
\int_{G}|\operatorname{tr} A(\varsigma \otimes \varsigma)(x)|^{2 s} d x \leq \frac{C}{d(\varsigma)^{2 s}}\left(\operatorname{tr} A A^{*}\right)^{s} .
$$

The most important consequence of this result is

(4.8) Theorem. Let $G$ be a connected compact group. Then any partial $m$-fold FTR set for $G$ is a $\Lambda(p)$ set for all $p \in(2, \infty)$.

Proof. We have seen already (Theorem (4.4)) that such sets are central $\Lambda(p)$ and so it suffices to prove they are local $\Lambda(p)$.

In view of the definition, the properties of FTR sets [5, 5.1], and the structure theorem (Theorem (4.1)) we need only establish the result in the case where $G=\prod_{\alpha \in A} S U\left(n_{\alpha}\right)$. Let $E \subseteq \widehat{G}$ be a partial $m$-fold FTR set for $G$, and fix a positive integer $s$. Let $A(m, s)=\left\{\alpha \in A: n_{\alpha} \leq 2 m s\right\}$, put $B=A \backslash A(m, s)$, and let $\mathscr{G}=\prod_{\alpha \in B} S U\left(n_{\alpha}\right)$. Then

$$
\sup \left\{d(\sigma): \sigma \in \bigcup_{j=0}^{m} \operatorname{FTR}^{j}\left(\prod_{\alpha \in A(m, s)} S U\left(n_{\alpha}\right)\right)\right\}<\infty,
$$

so $E$ is contained in a union of translates of

$$
D=\left\{\sigma \in \hat{\mathscr{G}}: \sigma \leq \gamma \text { for some } \gamma \in P_{m}\right\}
$$

by representations of bounded degree, where $P_{m}$ is as defined in (4.7). By (5.1) below it follows that $E$ is local $\Lambda(2 s)$ if $D$ is.

Consider $\sigma \in D$ and suppose $\sigma \leq \gamma \in P_{m}$. Let $\operatorname{tr} A \sigma \in \mathscr{T}_{\sigma}(\mathscr{G})$ and choose $U$ unitary so that $U \gamma U^{*}=\sigma \oplus \cdots$. Then using (4.7) we have

$$
\begin{aligned}
\int_{\mathscr{G}}|\operatorname{tr} A \sigma|^{2 s} & =\int_{\mathscr{G}}\left|\operatorname{tr}(A \oplus 0) U \gamma U^{*}\right|^{2 s} \\
& \leq \frac{C(s, m)}{d(\gamma)^{s}}\left(\operatorname{tr}\left|U^{*}(A \oplus 0) U\right|^{2}\right)^{s}=\frac{C(s, m)}{d(\gamma)^{s}}\left(\operatorname{tr} A A^{*}\right)^{s}
\end{aligned}
$$

so that

$$
\|\operatorname{tr} A \sigma\|_{2 s} \leq \frac{C(s, m)^{1 / 2 s}}{d(\sigma)^{1 / 2}}\left(\operatorname{tr} A A^{*}\right)^{1 / 2}=C(s, m)^{1 / 2 s}\|\operatorname{tr} A \sigma\|_{2}
$$

and $D$ is indeed local $\Lambda(2 s)$. 
Theorem (4.7) also has several amusing corollaries.

(4.9) Corollary. Let $\varsigma$ be the self-representation of either $U(n)$ or $S U(n)$ and let $m<n / 2$. Then any subrepresentation of $\varsigma^{m}$ has degree at least $O\left(n^{m}\right)$.

Proof. Let $\sigma \leq \varsigma^{m}$ be irreducible, and fix a unitary $U$ so that $U \varsigma^{m} U^{*}=$ $\sigma \oplus \cdots$. Applying Theorem (4.7) with $A=U^{*}(I \oplus 0) U$, where $I$ is a $d(\sigma) \times d(\sigma)$ identity matrix, yields

$$
\operatorname{tr} A A^{*} \geq \frac{n^{m}}{C(1, m)} \int|\operatorname{tr} \sigma|^{2}
$$

whence the result.

(4.10) Corollary. With the notation of (4.9), $\varsigma^{m}$ is not self-conjugate.

Proof. If $\varsigma^{m}$ were self-conjugate, then

$$
0 \neq \int(\operatorname{tr} \varsigma)^{2 m}=\sum_{i_{1}, \ldots, i_{2 m}} \int x_{i_{1} i_{1}} \cdots x_{i_{2 m} i_{2 m}} d x
$$

similar arguments to those in Lemma (4.6) show that each of the integrals in the sum is zero.

(4.11) Example. We give the prototype of a family of examples of non-Sidon sets containing representations of arbitrarily large degree which are nonetheless $\Lambda(p)$ sets for any $p \in(2, \infty)$. Let $\mathscr{G}=\prod_{n \geq 2} S U(n)$ and let $\pi_{n}: \mathscr{G} \rightarrow S U(n)$ denote the canonical projection. Then $E=\left\{\lambda_{2} \circ \pi_{n}\right\} \subseteq \operatorname{FTR}^{2}(\mathscr{G})$ is $\Lambda(p)$ for every $p \in(2, \infty)(4.8)$, but by $[5,5.5] E$ is not even a local Sidon set.

\section{EXISTENCE OF CENTRAL $\Lambda(p)$ SETS}

We collect together some estimates on the $\Lambda(p)$ constants for product groups.

(5.1) Lemma. Suppose $G=G_{1} \times G_{2}$ is a product of compact groups and that $E_{1} \subseteq \widehat{G}_{1}$, and $E_{2} \subseteq \widehat{G}_{2}$. Then

(i) $\eta_{p}\left(E_{1} \times E_{2}\right) \geq \eta_{p}\left(E_{1}\right) \eta_{p}\left(E_{2}\right)$,

(ii) $\eta_{p}^{0}\left(E_{1} \times E_{2}\right) \geq \eta_{p}^{0}\left(E_{1}\right) \eta_{p}^{0}\left(E_{2}\right)$, and

(iii) $\zeta_{p}\left(E_{1} \times E_{2}\right) \geq \zeta_{p}\left(E_{1}\right) \zeta_{p}\left(E_{2}\right)$.

For each $\tau \in E_{2}$ choose $\sigma_{\tau} \in \widehat{G}_{1}$. Put $E=\left\{\sigma_{\tau} \times \tau: \tau \in E_{2}\right\}$ and let $n=$ $\sup _{\tau \in E_{2}} d\left(\sigma_{\tau}\right)$. Then

(iv) $\eta_{p}(E) \leq \sqrt{2} n^{3 / 2} \eta_{p}\left(E_{2}\right)$,

(v) $\eta_{p}^{0}(E) \leq \sqrt{2} n^{3 / 2} \eta_{p}^{0}\left(E_{2}\right)$,

(vi) $\zeta_{p}(E) \leq \sqrt{2} n^{3 / 2} \zeta_{p}\left(E_{2}\right)$, and

(vii) in particular, $\sigma_{1} \times E_{2}$ is (local) (central) $\Lambda(p)$ if and only if $E_{2}$ is.

Proof. For (i), observe first that $\eta_{p}(E)=\sup \left\{\|f\|_{p}: f \in \mathscr{T}_{E}(G),\|f\|_{2}=1\right\}$. Let $\varepsilon>0$ and choose $f_{j} \in \mathscr{T}_{E_{j}}\left(G_{j}\right)$ so that $\left\|f_{j}\right\|_{2}=1$ and $\left\|f_{j}\right\|_{p} \geq \eta_{p}\left(E_{j}\right)-\varepsilon$. Then $f\left(x_{1}, x_{2}\right)=f_{1}\left(x_{1}\right) f_{2}\left(x_{2}\right) \in \mathscr{T}_{E_{1} \times E_{2}}(G)$, and since $\|f\|_{2}=1, \eta_{p}\left(E_{1} \times E_{2}\right) \geq$ $\|f\|_{p}=\left\|f_{1}\right\|_{p}\left\|f_{2}\right\|_{p} \geq\left(\eta_{p}\left(E_{1}\right)-\varepsilon\right)\left(\eta_{p}\left(E_{2}\right)-\varepsilon\right)$; since $\varepsilon$ is arbitrary, (i) follows. Parts (ii) and (iii) are proved similarly.

For part (iv) the inequality is trivial if $n$ is infinite, so we suppose $n<\infty$. 
Let $n_{\tau}=d\left(\sigma_{\tau}\right)$, and consider $f=\sum_{\tau \in E_{2}} \operatorname{tr} A_{\tau}\left(\sigma_{\tau} \times \tau\right) \in \mathscr{T}_{E}(G)$ : we can write $A_{\tau}=\sum_{i, j=1}^{n_{\tau}} B_{i j}^{(\tau)} \otimes C_{i j}^{(\tau)}$, where $B_{i j}^{(\tau)}$ is an $n_{\tau} \times n_{\tau}$ matrix having a 1 in the $(i, j)$ position and zeros everywhere else, and $C_{i j}^{(\tau)}$ is a $d(\tau) \times d(\tau)$ matrix. Observe that $\operatorname{tr} B_{i j}^{(\tau)} \sigma_{\tau}\left(x_{1}\right)=\sigma_{\tau}\left(x_{1}\right)_{j i}$ and put $D_{\tau}\left(x_{1}\right)=\sum_{i, j=1}^{n_{\tau}} \sigma_{\tau}\left(x_{1}\right)_{j i} C_{i j}^{(\tau)}$; since $\sigma_{\tau}\left(x_{1}\right)$ is unitary its entries have modulus at most 1 , so that

$$
\operatorname{tr} D_{\tau}\left(x_{1}\right) D_{\tau}\left(x_{1}\right)^{*} \leq 2 n_{\tau}^{2} \sum_{i j=1}^{n_{\tau}} \operatorname{tr} C_{i j}^{(\tau)} C_{i j}^{(\tau) *}
$$

for any $x_{1} \in G_{1}$. Hence

$$
\begin{aligned}
\|f\|_{p}^{p} & =\int_{G_{1}} \int_{G_{2}}\left|\sum_{\tau \in E_{2}} \operatorname{tr} D_{\tau}\left(x_{1}\right) \tau\left(x_{2}\right)\right|^{p} d x_{2} d x_{1} \\
& \leq \int_{G_{1}}\left(\eta_{p}\left(E_{2}\right)\right)^{p}\left\|\sum_{\tau \in E_{2}} \operatorname{tr} D_{\tau}\left(x_{1}\right) \tau\right\|_{2}^{p} d x_{1} \\
& =\left(\eta_{p}\left(E_{2}\right)\right)^{p} \int_{G_{1}}\left(\sum_{\tau \in E_{2}} \frac{\operatorname{tr} D_{\tau}\left(x_{1}\right) D_{\tau}\left(x_{1}\right)^{*}}{d(\tau)}\right)^{p / 2} d x_{1}
\end{aligned}
$$

so that

$$
\begin{aligned}
\|f\|_{p} & \leq \eta_{p}\left(E_{2}\right) \sqrt{2} n\left(\sum_{\tau \in E_{2}} \sum_{i, j=1}^{n_{\tau}} \frac{\operatorname{tr} C_{i j}^{(\tau)} C_{i j}^{(\tau) *}}{d(\tau)}\right)^{1 / 2} \\
& \leq \eta_{p}\left(E_{2}\right) \sqrt{2} n^{3 / 2}\left(\sum_{\tau \in E_{2}} \frac{\operatorname{tr} A_{\tau} A_{\tau}^{*}}{d\left(\sigma_{\tau} \times \tau\right)}\right)^{1 / 2}=\eta_{p}\left(E_{2}\right) \sqrt{2} n^{3 / 2}\|f\|_{2}
\end{aligned}
$$

and (iv) holds.

Parts (v) and (vi) are proved similarly, and (vii) is immediate from the preceding parts.

(5.2) Lemma. Let $G$ be a compact group and let $\sigma \in \widehat{G}$. Suppose $\sigma \otimes \sigma$ can be decomposed into irreducibles as $\bigoplus_{j=1}^{M} m_{j} \tau_{j}$, and that $\sigma \otimes \bar{\sigma}$ decomposes as $\bigoplus_{j=1}^{N} n_{j} \tau_{j}^{\prime}$. Then $\left(\zeta_{4}^{0}(\sigma)\right)^{4}=\sum_{j=1}^{M} m_{j}^{2}=\sum_{j=1}^{N} n_{j}^{2}$.

Proof. Observe that $\zeta_{4}(\sigma)=\zeta_{4}^{0}(\sigma)=\|\operatorname{tr} \sigma\|_{4}$, and that

$$
\|\operatorname{tr} \sigma\|_{4}^{4}=\int_{G}|\operatorname{tr} \sigma \otimes \sigma|^{2}=\int_{G}|\operatorname{tr} \sigma \otimes \bar{\sigma}|^{2}
$$

now apply $[9,27.31]$.

We now describe all local central $\Lambda(4)$ sets on certain product groups. Our description parallels strongly that for local Sidon sets on product groups given by Cartwright and McMullen [5, 5.5].

(5.3) Proposition. Let $\mathscr{G}=\prod_{\alpha \in A} G_{\alpha}$ where each $G_{\alpha}$ is a compact connected almost simple Lie group of rank $l_{\alpha}$, and suppose $E \subseteq \hat{\mathscr{G}}$ is local central $\Lambda(4)$. 
Then there exist a partition $A=A_{1} \cup A_{2} \cup A_{3}$ of $A$, subsets $E_{j} \subseteq \widehat{G}_{j}$, the dual of $G_{j}=\prod_{\alpha \in A_{j}} G_{\alpha}(j=1,2,3)$, and $M \in \mathbb{N}$ such that

(i) $E \subseteq E_{1} \times E_{2} \times E_{3}$,

(ii) $E_{1}=\{1\}$,

(iii) $\sup \left\{l_{\alpha}: \alpha \in A_{2}\right\}<\infty$ and $\sup \left\{d(\sigma): \sigma \in E_{2}\right\}<\infty$, and

(iv) $G_{3}$ has no exceptional factors, and $E_{3}=\operatorname{FTR}^{M-1}\left(G_{3}\right) \cup \operatorname{FTR}^{M}\left(G_{3}\right)$.

Conversely, any set satisfying (i)-(iv) is local central $\Lambda(p)$ for all $p \in(2, \infty)$. Proof. Choose $m \geq\left(\zeta_{4}^{0}(E)\right)^{4}$, and put $A_{1}=\left\{\alpha \in A: \sigma_{\alpha}\right.$ is trivial for every $\sigma \in$ $E\}$. Let $A_{2}=\left\{\alpha \in A \backslash A_{1}: l_{\alpha} \leq \max (8,2 m)\right\}$, put $A_{3}=A \backslash\left(A_{1} \cup A_{2}\right)$, and let $E_{j}=\left\{\left(\sigma_{\alpha}\right)_{\alpha \in A_{j}}:\left(\sigma_{\alpha}\right)_{\alpha \in A} \in E\right\}$ for $j=1,2,3$. Then (i), (ii), and the first part of (iii) hold by construction.

It is easy to see that $\zeta_{4}^{0}\left(\left(\sigma_{\alpha}\right)\right)=\prod_{\alpha} \zeta_{4}^{0}\left(\sigma_{\alpha}\right)$ and that if $d(\sigma)>1$ then $\left(\zeta_{4}^{0}(\sigma)\right)^{4} \geq 2$. Since $G_{\alpha}$ is almost simple, $d\left(\sigma_{\alpha}\right)>1$ if and only if $\sigma_{\alpha}$ is nontrivial. Hence each $\sigma \in E$ has at most $\log _{2} m$ nontrivial components.

Denote by $\lambda_{\alpha}=\sum_{k=1}^{l_{\alpha}} m_{k} \lambda_{k}$ the highest weight of $\sigma_{\alpha}$. By Lemma (3.2), $\sigma_{\alpha} \otimes \sigma_{\alpha}$ contains $2 \lambda_{\alpha}-j \alpha_{k}, 0 \leq j \leq m_{k}$, for each $1 \leq k \leq l_{\alpha}$. For $j>0$ these are all distinct, so $\left(\zeta_{4}^{0}\left(\sigma_{\alpha}\right)\right)^{4} \geq \sum_{k=1}^{l_{\alpha}} m_{k}$. In particular, the coefficients $m_{k}$ are bounded by $m$; it follows from the Weyl dimension formula [11, Corollary 24.3] that $d\left(\sigma_{\alpha}\right) \leq d\left(m \delta_{\alpha}\right)=(m+1)^{\left|\Phi_{\alpha}^{+}\right|}$(where $\delta_{\alpha}=\sum_{k=1}^{l_{\alpha}} \lambda_{k}$, and $\Phi_{\alpha}$ is the root system for $\left.G_{\alpha}\right)$.

Consider $\sigma=\left(\sigma_{\alpha}\right)_{\alpha \in A_{2}} \in E_{2}$ : it has at most $\log _{2} m$ nontrivial components, each of which has degree at most that of $m \delta_{\alpha}$; since $l_{\alpha} \leq \max (8,2 m)$ it follows from the classification theorem and [17] that $\left|\Phi_{\alpha}^{+}\right| \leq \max \left(120,4 m^{2}\right)$. Thus $d(\sigma) \leq(m+1)^{\max \left(120,4 m^{2}\right) \log _{2} m}$ and (iii) holds.

Lastly, consider $\sigma=\left(\sigma_{\alpha}\right)_{\alpha \in A_{3}} \in E_{3}$. Observe that since $l_{\alpha}>8$, none of the $G_{\alpha}$ is exceptional. From Lemmas (3.4) and (5.2) applied to $\sigma_{\alpha}$, it follows that $m \geq N_{\alpha}+1$, where $N_{\alpha}$ is the $N$ of Lemma (3.4). If $G_{\alpha}$ is of type $B$ or $C$ then the coefficients of $\lambda_{\alpha}$ are zero for $k>N_{\alpha}$ and hence for $k>m$; since $l_{\alpha}>2 m$ the same holds for $G_{\alpha}$ of type $D$. For $G_{\alpha}$ of type $A$ the coefficients are zero for $N_{\alpha}<k \leq l_{\alpha}-N_{\alpha}$ and so for $m<k \leq l_{\alpha}-m$. For $G_{\alpha}$ of type $B$, $C$ or $D$ we have $\lambda_{j} \leq \lambda_{1}^{j}$ for $1 \leq j<l_{\alpha}-1$ (at least), and for type $A$ we have both $\lambda_{j} \leq \lambda_{1}^{j}$ and $\lambda_{j} \leq\left(\overline{\lambda_{1}}\right)^{l_{\alpha}+1-j}$ for $1 \leq j \leq l_{\alpha}$. Hence in types $B, C$, and $D$ we have $\sigma_{\alpha} \leq \lambda_{1}^{J_{\alpha}}$ where $J_{\alpha}=\sum_{k=1}^{m} k m_{k} \leq m^{2}$, since, as noted earlier, the sum of the coefficients is bounded by $m$. In type $A$ we have $\sigma_{\alpha} \leq \lambda_{1}^{J_{\alpha}} \otimes\left(\overline{\lambda_{1}}\right)^{K_{\alpha}}$ where $J_{\alpha}=\sum_{k=1}^{m} k m_{k}, K_{\alpha}=\sum_{k=1}^{m} k m_{l_{\alpha}+1-k}$, and $J_{\alpha}+K_{\alpha} \leq m^{2}$. Thus $\sigma_{\alpha}$ belongs to $\operatorname{FTR}^{j}\left(G_{\alpha}\right)$ for some $j \leq m^{2}$. Further, since $\sigma$ has at most $\log _{2} m$ nontrivial components, it follows that $\sigma \in \operatorname{FTR}^{j}\left(G_{3}\right) \subseteq \operatorname{FTR}^{j+2}\left(G_{3}\right) \subseteq \cdots$ for some $j \leq m^{2} \log _{2} m$. If we now choose $M \geq m^{2} \log _{2} m$ and redefine $E_{3}$ to be FTR $^{M-1}\left(G_{3}\right) \cup$ FTR $^{M}\left(G_{3}\right)$, then (i)-(iv) hold as claimed.

The converse follows from Theorem (4.4) and Lemma (5.1).

A corollary of Cartwright and McMullen's result on local Sidon sets is that tall connected compact groups have the property that every local Sidon set is in fact Sidon. A similar situation obtains for local central $\Lambda(4)$ sets, in startling contrast to the abelian case:

(5.4) Corollary. Let $G$ be a tall connected compact group and suppose $E \subseteq \widehat{G}$ is a local central $\Lambda(4)$ set. Then $E$ is central $\Lambda(p)$ for all $p \in(2, \infty)$. 
Proof. Let $\pi: \mathscr{G} \rightarrow G$ be the standard structure theorem epimorphism; then $\mathscr{G}$ is also tall and has the form of Proposition (5.3). Applying (5.3) to $E \circ \pi$ we see that $E_{2}$ must be finite by tallness; thus by Lemma (5.1) and Theorem (4.4) $E_{1} \times E_{2} \times E_{3}$, and hence $E$, is central $\Lambda(p)$ for all $p>2$.

We can now give a characterization of the compact connected groups which admit an infinite central $\Lambda(4)$ set; this should be compared with Cartwright and McMullen's result [5, 6.2] for Sidon sets.

(5.5) Theorem. Let $G$ be a connected compact group and $4 \leq p<\infty$. Then the following are equivalent:

(i) $G$ has an infinite central $\Lambda(p)$ set.

(ii) $G$ has an infinite local central $\Lambda(4)$ set.

(iii) Either $G$ is not tall, or $G$ is tall and is not a Lie group.

Proof. Clearly (i) implies (ii). Suppose $E \subseteq \widehat{G}$ is an infinite local central $\Lambda(4)$ set and that $G$ is tall. Then $G$ is a quotient of $\mathscr{G}=\prod_{\alpha \in A} G_{\alpha}$, with each $G_{\alpha}$ an almost simple simply-connected compact Lie group. It follows from (5.3) and (4.2) that $\mathscr{G}=\mathscr{G}_{1} \times \mathscr{G}_{2}$ where $\mathscr{G}_{2}$ has no exceptional factors, and (by tallness of $G) \mathscr{G}_{2}$ has an infinite $m$-fold FTR set. In particular, $\mathscr{G}_{2}$ has infinitely many factors, so $[15,6.4 .5] G$ is not a Lie group; thus (ii) implies (iii).

Since $[12,2.4]$ nontall groups admit infinite Sidon sets, to prove (iii) implies (i) we need only consider the case $G$ tall and not Lie. Then there is an epimorphism $\pi: \mathscr{G} \rightarrow G$ where $\mathscr{G}=\prod_{\alpha \in A} G_{\alpha}$; here the $G_{\alpha}$ are as usual and $A$ is infinite. Since $\operatorname{ker} \pi$ is central, there is an epimorphism $\pi^{\prime}$ of $G$ onto $\mathscr{G}^{\prime}=\mathscr{G} / Z(\mathscr{G})=\prod_{\alpha \in A} G_{\alpha} / Z\left(G_{\alpha}\right)$. Let $A^{\prime}=\left\{\alpha \in A: G_{\alpha}\right.$ is nonexceptional $\}$; by tallness $A^{\prime}$ is also infinite. For $\alpha \in A^{\prime}$ let $\tau_{\alpha}$ denote the representation of $G_{\alpha}$ whose highest weight is $\lambda_{2}$ if $G_{\alpha}$ is of type $B, C$, or $D$, or whose highest weight is $\lambda_{1}+\lambda_{l_{\alpha}}$ if $G_{\alpha}$ is of type $A_{l_{\alpha}}$. Then [17] $\operatorname{ker} \tau_{\alpha}=Z\left(G_{\alpha}\right)$, so we may think of $\tau_{\alpha}$ as being a representation of $G_{\alpha}^{\prime}=G_{\alpha} / Z\left(G_{\alpha}\right)$. If we denote by $\pi_{\alpha}^{\prime}: \mathscr{G}^{\prime} \rightarrow G_{\alpha}^{\prime}$ the canonical epimorphism, then $E^{\prime}=\left\{\tau_{\alpha} \circ \pi_{\alpha}^{\prime}: \alpha \in A^{\prime}\right\}$ is contained in $\operatorname{FTR}^{2}\left(\mathscr{G}^{\prime}\right)$ and so (4.4) is an infinite central $\Lambda(p)$ set in $\widehat{\mathscr{G}}^{\prime}$ for any $p \in(2, \infty)$, and hence $E^{\prime} \circ \pi^{\prime}$ is an infinite central $\Lambda(p)$ set in $\widehat{G}$.

\section{EXISTENCE OF $\Lambda(p)$ SETS}

(6.1) Proposition. Let $\mathscr{G}=\prod_{\alpha \in A} G_{\alpha}$ be a product of compact groups, and let $E \subseteq \widehat{\mathscr{G}}$. Suppose that each $\left(\sigma_{\alpha}\right)_{\alpha \in A} \in E$ has at most one nontrivial factor, and that for each $\alpha \in A$ there is at most one element of $E$ which is nontrivial on $G_{\alpha}$. Let $p \in(2, \infty)$. Then $E$ is a (central) $\Lambda(p)$ set if and only if $E$ is a local (central) $\Lambda(p)$ set.

Proof. For even integers $p$ the result follows from [1, $\S 5]$.

Suppose $E$ is a local $\Lambda(p)$ set; without loss of generality we may suppose that $E$ does not contain the trivial representation. Let $f=\sum_{\sigma \in E} d(\sigma) \operatorname{tr} A_{\sigma} \sigma \in$ $\mathscr{T}_{E}(G)$; if we put $\left\{\sigma_{1}, \ldots, \sigma_{N}\right\}=\left\{\sigma \in E: A_{\sigma} \neq 0\right\}$ then we may write $f=\sum_{i=1}^{N} d_{i} \operatorname{tr} A_{i} \sigma_{i}$. The special form of the representations in $E$ imply that $\left\{d_{i} \operatorname{tr} A_{i} \sigma_{i}\right\}_{i=1}^{N}$ is a collection of independent random variables. Hence, since $\int d_{i} \operatorname{tr} A_{i} \sigma_{i}=0,\left\{\sum_{i=1}^{n} d_{i} \operatorname{tr} A_{i} \sigma_{i}\right\}_{n \geq 1}$ is a martingale. 
By Burkholder's theorem on the square function of the martingale difference series [4, Theorem 9], there exists a constant $C_{p}$, not depending on $f$, such that

$$
\begin{aligned}
\|f\|_{p} & \leq C_{p}\left\|\left(\sum_{i=1}^{N}\left|d_{i} \operatorname{tr} A_{i} \sigma_{i}\right|^{2}\right)^{1 / 2}\right\|_{p} \leq C_{p}\left(\sum_{i=1}^{N}\left\|d_{i} \operatorname{tr} A_{i} \sigma_{i}\right\|_{p}^{2}\right)^{1 / 2} \\
& \leq C_{p} \eta_{p}^{0}(E)\left(\sum_{i=1}^{N}\left\|d_{i} \operatorname{tr} A_{i} \sigma_{i}\right\|_{2}^{2}\right)^{1 / 2}=C_{p} \eta_{p}^{0}(E)\|f\|_{2} .
\end{aligned}
$$

Thus $E$ is a $\Lambda(p)$ set. A similar proof covers the local central case.

The reverse implications are obvious.

(6.2) Theorem. Let $G$ be a compact connected group, and let $4 \leq p<\infty$. Any infinite local $\Lambda(p)$ set on $G$ contains an infinite $\Lambda(p)$ set.

Proof. Without loss of generality we may suppose that $G=T \times \mathscr{G}$, where $T$ is a connected compact abelian group and $\mathscr{G}=\prod_{\alpha \in A} G_{\alpha}$, with each $G_{\alpha}$ being an almost simple simply-connected compact Lie group. Each $\sigma \in \widehat{G}$ can be written in the form $\sigma_{T} \times\left(\sigma_{\alpha}\right)_{\alpha \in A}$, where $\sigma_{T}$ is a character of $T$ and $\sigma_{\alpha} \in \widehat{G_{\alpha}}$, with only finitely many of the $\sigma_{\alpha}$ being nontrivial. Suppose $E \subseteq \widehat{G}$ is an infinite local $\Lambda(p)$ set.

If $E_{\mathscr{G}}=\left\{\left(\sigma_{\alpha}\right)_{\alpha \in A}: \sigma \in E\right\}$ is finite then there is $\tau \in \hat{\mathscr{G}}$ so that $\sigma_{T} \times \tau \in E$ for infinitely many $\sigma_{T} \in \widehat{T}$. It follows from $[9,37.18]$ that $\left\{\sigma_{T} \in \widehat{T}: \sigma_{T} \times \tau \in\right.$ $E\}$, being infinite, contains an infinite Sidon, and hence $\Lambda(p)$, set $E_{T}$. Then (Lemma (5.1)) $E_{T} \times \tau$ is an infinite $\Lambda(p)$ subset of $E$.

Otherwise, $E_{\mathscr{G}}$ must be an infinite local $\Lambda(p)$ subset of $\hat{\mathscr{G}}$ (Lemma (5.1)). For each $\sigma=\left(\sigma_{\alpha}\right)_{\alpha \in A} \in E_{\mathscr{G}}$, let $A_{\sigma}=\left\{\alpha \in A: \sigma_{\alpha} \neq 1\right\}$, and note that $\sup _{\sigma \in E_{\mathscr{G}}}\left|A_{\sigma}\right| \leq 4 \log _{2}\left(\zeta_{4}^{0}\left(E_{\mathscr{G}}\right)\right)<\infty$. If $E_{\mathscr{G}}$ contains an infinite subset $E_{\mathscr{G}}^{\prime}$ so that the $A_{\sigma}, \sigma \in E_{\mathscr{G}}^{\prime}$, are disjoint, then by (6.1) $E_{\mathscr{G}}^{\prime}$ is $\Lambda(p)$. For each $\sigma \in E_{\mathscr{G}}^{\prime}$ choose one $\sigma_{T} \in \widehat{T}$ so that $\sigma_{T} \times \sigma \in E$; then by Lemma (5.1) $\left\{\sigma_{T} \times \sigma: \sigma \in E_{\mathscr{G}}^{\prime}\right\}$ is an infinite $\Lambda(p)$ subset of $E$.

If no $E_{\mathscr{G}}^{\prime}$ as above exists, then there must be an index $\alpha_{0}$ and an infinite subset $E_{\mathscr{G}}^{\prime} \subseteq E_{\mathscr{G}}$ so that $\alpha_{0} \in A_{\sigma}$ for every $\sigma \in E_{\mathscr{G}}^{\prime}$. Now (5.3) implies that there are only a finite number of possibilities for $\sigma_{\alpha_{0}}$, and hence there is an infinite subset of $E_{\mathscr{G}}^{\prime}$ whose elements agree on $G_{\alpha_{0}}$, that is, having the form $\sigma_{0} \times E_{\mathscr{G}}^{\prime \prime}$. By (5.1), $E_{\mathscr{G}}^{\prime \prime}$ is an infinite local $\Lambda(p)$ subset in the dual of $\mathscr{G}^{\prime \prime}=\prod_{\alpha \in A^{\prime}} G_{\alpha}$ where $A^{\prime}=A \backslash\left\{\alpha_{0}\right\}$, and again by (5.1) any infinite $\Lambda(p)$ subset of $E_{\zeta}^{\prime \prime}$ will yield an infinite $\Lambda(p)$ subset of $E$. Our construction ensures that $\left|A_{\sigma}^{\prime}\right|=\left|A_{\sigma_{0} \times \sigma}\right|-1$ for $\sigma \in E_{\mathscr{G}}^{\prime \prime}$; if we now repeat the above arguments the worst that can happen is that we reach the case of an infinite local $\Lambda(p)$ set each of whose elements is supported by a single $G_{\alpha}$. Since no $G_{\alpha}$ admits an infinite local $\Lambda(4)$ set [6], there is an infinite subset to which (6.1) can be applied, and we are done.

(6.3) Remark. Theorems (4.8) and (6.2) bring us close to an analogue of (5.5) for $\Lambda(p)$ sets. The chief obstruction is in determining whether the set $E^{\prime}$ in 
the proof of (5.5) is local $\Lambda(p)$ when the ranks of the $G_{\alpha}$ are unbounded. The representations in $E^{\prime}$ are less "rank-independent" than those which occur in FTR or partial $m$-fold FTR sets in the following sense: the highest weight of each representation in $E^{\prime}$ has a weight whose multiplicity increases with the rank of $G_{\alpha}$ (for example, 0 occurs as a weight of $\lambda_{1}+\lambda_{l_{\alpha}}$ with multiplicity $l_{\alpha}$ ) whereas the representations in FTR or partial $m$-fold FTR sets have the multiplicities of their weights bounded independently of the ranks of the supporting groups. We have been unable to determine whether this difference, or any other, is sufficient to prevent $E^{\prime}$ being local $\Lambda(4)$.

\section{REFERENCES}

1. G. Benke, On the hypergroup structure of central $\Lambda(p)$ sets, Pacific J. Math. 50 (1974), 19-27.

2. A. Bonami, Étude des coefficients de Fourier des fonctions de $L^{p}(G)$, Ann. Inst. Fourier (Grenoble) 20 (1970), 335-402.

3. N. Bourbaki, Groupes et algèbres de Lie, Hermann, Paris, 1975.

4. D. L. Burkholder, Martingale transforms, Ann. Math. Statist. 37 (1966), 1494-1504.

5. D. I. Cartwright and J. R. McMullen, $A$ structural criterion for the existence of infinite Sidon sets, Pacific J. Math. 96 (1981), 301-317.

6. C. Cecchini, Lacunary Fourier series on compact Lie groups, J. Funct. Anal. 11 (1972), 191-203.

7. A. H. Dooley, Norms of characters and lacunarity for compact Lie groups, J. Funct. Anal. 32 (1979), 254-267.

8. A. Figà-Talamanca and D. Rider, A theorem of Littlewood and lacunary series for compact groups, Pacific J. Math. 16 (1966), 505-514.

9. E. Hewitt and K. A. Ross, Abstract harmonic analysis. II, Springer-Verlag, New York, Heidelberg and Berlin, 1970.

10. K. E. Hare, $L^{p}$-improving measures on compact non-abelian groups, J. Austral. Math. Soc. Ser. A 46 (1989), 402-414.

11. J. E. Humphreys, Introduction to Lie algebras and representation theory, Springer-Verlag, New York, Heidelberg and Berlin, 1972.

12. M. F. Hutchinson, Non-tall compact groups admit infinite Sidon sets, J. Austral. Math. Soc. Ser. A 23 (1977), 467-475.

13. A. W. Knapp, Representation theory of semisimple groups, Princeton Univ. Press, Princeton, N.J., 1986.

14. J. M. López and K. A. Ross, Sidon sets, Lecture Notes in Pure and Appl. Math., no. 13, Marcel Dekker, New York, 1975.

15. J. F. Price, Lie groups and compact groups, London Math. Soc. Lecture Note Ser., no. 25, Cambridge Univ. Press, Cambridge, London, New York and Melbourne, 1977.

16. D. Rider, Central lacunary sets, Monatsh. Math. 76 (1972), 328-338.

17. J. Tits, Tabellen zu den einfachen Lie gruppen und ihren darstellungen, Lecture Notes in Math., vol. 40, Springer-Verlag, Berlin, Heidelberg and New York, 1967.

18. D. C. Wilson, A general criterion for the existence of infinite Sidon sets, J. Austral. Math. Soc. Ser. A 45 (1988), 11-29.

Department of Pure Mathematics, University of Waterloo, Waterloo, Ontario, CANADA N2L 3G1

E-mail address: kehare@watmath.uwaterloo.ca

School of Mathematics, University of New South Wales, PO Box 1, Kensington, New South Wales 2033, Australia

E-mail address: d.wilson@unsw.edu.au 